1314 EFFECT OF BLOOD TRANSFUSION ON LIPID PEROXIDATION PRODUCTS IN SICK NEONATES - AN EXPLORATORY STUDY

doi:10.1136/archdischild-2012-302724.1314

${ }^{1} \mathrm{~K}$ Mukhopadhyay, ${ }^{2} \mathrm{M}$ Ali, ${ }^{2} \mathrm{~S}$ Bhattacharya, ${ }^{2} \mathrm{P}$ Kumar. ${ }^{1}$ Neonatal Unit, Dept of Pediatrics; 2PGIMER, Chandigarh, India

Background and Aims Preterm neonates have lower anti oxidant defense system and repeated blood transfusion may further increase the oxidative stress specially in sick preterm neonates. The aim was to assess the effect of blood transfusion on lipid peroxidation product Malondialdehyde (MDA) in sick neonates.

Methods This was an exploratory study in a Level III neonatal unit in which 50 consecutive sick neonates of $\leq 34$ weeks gestation were enrolled who received blood transfusion till day 28. Primary outcomes were

1. Pre and post transfusion blood and Urine MDA and

2. Pre transfusion SOD and Catalase. Secondary outcome was Pretransfusion MDA and antioxidant enzymes in various neonatal morbidities.

Results The mean birth weight and gestational age were $1416 \pm 219$ grams and $29.9 \pm 2.5$ weeks respectively. The pre transfusion blood and urine MDA were $4.2 \pm 1.2 \mathrm{nmol} / \mathrm{ml}$ and $6.96 \pm 3.7 \mathrm{nmol} / \mathrm{ml}$ respectively. These levels increased significantly from baseline after each transfusion. Base line MDA was higher $(p=0.032)$ and SOD $(p=0.02)$ and Catalase $(p=0.00)$ were significantly lower in babies $<30$ wks gestation.

Pre transfusion Blood MDA levels were significantly higher $(p=0.00)$ in the babies who had IVH and BPD while urine MDA was significantly high in BPD babies. SOD and Catalase levels were significantly $(p=0.00)$ lower in babies who developed BPD

Conclusion Baseline oxidant levels are higher and anti- oxidant enzymes are lower in in $<30$ weeks gestation babies and in those who developed BPD and IVH. Blood transfusion further increases lipid peroxidation products.

\section{MATERNAL PREFERENCES WITH REGARD TO INDOMETHACIN PROPHYLAXIS VS TREATMENT OPTIONS IN PRETERM INFANTS}

doi:10.1136/archdischild-2012-302724.1315

'KM AlFaleh, 'A Al Osaimi, 'E Alluwaimi, 'S AlRajebah, 'B Al Otaibi, 'F AlRasheed, ${ }^{2} \mathrm{~S}$ AlAlaiyan. 'Pediatrics, King Saud University; ${ }^{2}$ Pediatrics, KFSH \& RC, Riyadh, Saudi Arabia

Background Indomethacin prophylaxis reduces important short term outcomes in ELBW infants, however with no effect on BPD or long term neurosensory impairment. Neonatologists are diverse with regard to the utilization of a prophylactic versus treatment strategies in the management of high risk ELBW infants.

Objectives To elicit maternal preferences with regard to indomethacin prophylaxis versus treatment options in ELBW infants utilizing a decision aid tool.

Methods Pregnant women at 23-28 weeks gestation, women of high risk pregnancy and mothers of admitted ELBW infants were enrolled. A computer based interactive decision aid (DA) tool was utilized during interviews. In the first part, the DA provided information with regard to prematurity and various morbidities affecting the preterm infants, then detailed information of pros and cons and prophylactic versus treatment options. In the second part, it coached participants in clarifying values and preferences.

Results Two hundred ninety nine participants were enrolled. Of those; $75 \%$ were pregnant women at 23 to 28 weeks, $19 \%$ were pregnant of high risk pregnancy and $6 \%$ recently had an ELBW infant. $82 \%$ of enrolled women preferred a prophylactic indomethacin strategy versus treatment in the management of their infants. When asked about their values, the occurrence of IVH was rated lowest among all other neonatal morbidities affecting the preterm infant.

Conclusion In contrast to neonatal practitioners, mothers strongly preferred an indomethacin prophylactic strategy in the management of preterm infants.

\section{DIAGNOSTIC ROLE OF CALPROTECTIN IN NEWBORNS}

doi:10.1136/archdischild-2012-302724.1316

${ }^{1} \mathrm{G}$ Vida, 'T Lévai, ${ }^{2} \mathrm{~A}$ Peti, ${ }^{1} \mathrm{~J}$ Gyarmati, 'S Funke, ${ }^{1} \mathrm{~T}$ Ertl, ${ }^{2} \mathrm{GL}$ Kovács. 'Department of Obstetrics and Gynecology; ${ }^{2}$ Department of Laboratory Medicine, University of Pécs, Pécs, Hungary

Introduction Calprotectin is a member of the S100 protein family binding calcium-and zinc and has been demonstrated as one of the most sensitive marker of the intestinal inflammation. It is secreted into the faeces by neutrophil granulocytes and can non-invasively be monitored neonatal infectious disease.

Background and Aims Determining the physiological age-related changes of calprotectin level in faeces and describing the measured values in pathological conditions. Exploring the influential factors of the calprotectin concentration and establishing a reference value regarding neonatal intestinal diseases.

Methods 106 stool samples were collected from 66 newborn (51/15: mature/premature) in the University of Pecs followed by calprotectin determination performed by ELISA. Throughout the study, the calprotectin content of the normal meconium was monitored and data were processed retrospectively.

Results The faecal calprotectin concentration of mature infants is many times higher than the literature indicated four year reference value. This biomarker level showed typical changes during the first week of life (median: $286.94 \mathrm{ug} / \mathrm{g}$ ). Lower calprotectin levels were found in infants whose physiological weight loss had stopped in the first three days. Furthermore, changes in calprotectin level occured later after caesarean section than after vaginal delivery and higher concentrations were found in infants after breast-feeding compared to formula-feeding. Moreover, high calprotectin levels (646.2 and $1243.2 \mathrm{ug} / \mathrm{g}$ ) had been detected before manifestation of symptoms in two lethal necrotizing enterocolitis associated cases.

Conclusions Determination of faecal calprotectin is considered as a useful prognostic biomarker in premature infants showing symptoms, such as intestinal distress or general infection.

\section{ARM FUNCTIONS IN CHILDREN WITH A CONSERVATIVELY TREATED OBSTETRIC BRACHIAL PLEXUS PALSY}

doi:10.1136/archdischild-2012-302724.1317

${ }^{1} \mathrm{~K}$ de Boer, ${ }^{2} \mathrm{JD}$ Stenvers, ${ }^{3} \mathrm{WB}$ Geven, ${ }^{4} \mathrm{LD}$ Jelsma, ${ }^{5} \mathrm{AS}$ Niemeijer. ${ }^{1}$ Physical Therapy, Martini Hospital Groningen; Groningen; 2 Physiotherapie Haren, Haren; ${ }^{3 P e d i a t r i c s, ~}$ Martini Hospital Groningen; Groningen; "Physiotherapie Paterswolde, Paterswolde; ${ }^{5}$ Avans, Breda, The Netherlands

Aim To determine whether conservative treatment of children with Obstetrics Plexus Brachialis Palsy (OBPP) results in restrictions of activities, participation and body functions and structure.

Method 22 children, born with OBPP were examined at the age of 4 till 17 years. The activity level is assessed by the Movement Assessment Battery for Children-2 (M-ABC 2) and the Bruininks-Oseretsky Test of Motor Proficiency, Second Edition (BOT-2). For the Body functions and structure, the muscle strength is measured by the Hand Held Dynamometer (HHD) and the joint mobility by goniometry and the Stenvers tests. In addition the scapula position is observed. The Participation is documented by Children's Assessment of Participation and Enjoyment (CAPE).

Results Concerning the body functions and structure we found differences between the arms in joint mobility and muscle strength. $45.5 \%$ of the children have a medio-rotation of the scapula. These 
differences lead to a different movement pattern of the affected arm. The effect of the OBPP on the level of activity and participation seems to be of no importance. The children with an OBPP do not stay behind with children of their age in physical and social activities.

Conclusions Children with a conservative treaded OBPP have persisting restrictions specially on the level of body functions and structure. Despite these restrictions there is no relevant effect shown on the activity and participation level. Using the mobility tests we think there is a relation between position of the scapula and the joint mobility.

\section{SMOKING AND OTHER PRE-GESTATIONAL RISK FACTORS FOR SPONTANEOUS PRETERM BIRTH}

doi:10.1136/archdischild-2012-302724.1318

1.2PF Freitas, ${ }^{3}$ E Grillo. ${ }^{1}$ Public Health, Universidade Federal de Santa Catarina; ${ }^{2}$ Curso de Medicina, Universidade do Sul de Santa Catarina UNISUL; ${ }^{3 P e d i a t r i a, ~ U n i v e r s i d a d e ~}$ Federal de Santa Catarina, Florianópolis, Brazil

Objectives To investigate pre-gestational risk factors for spontaneous preterm birth and, the role of smoking and its cumulative effects on prematurity.

Methods A case-control study analyzed all births $(2,198)$ occurring in a tertiary maternity hospital between April 2002 and July 2004. Spontaneous preterm births of single and live newborns without malformations were selected as cases. Controls were all the term births of live and single newborns without malformations during the same period. Three outcomes were studied: all preterm births ( $<37$ weeks), less than 35 weeks and less than 32 weeks of gestational age. Logistic regression was used to obtain the independent effect of pre-gestational risk factors.

Results Maternal age of less than 20 years, low schooling, low maternal pre-gestational body mass index and smoking showed significant, independent association with spontaneous preterm birth for the three outcomes. For all these risk factors, excepting maternal smoking, odds ratios increased with decreasing gestational age at birth and this trend was significant for low maternal age and low pre-gestational body mass index.

Conclusions The cumulative effects of smoking calls for the need to encourage smoking cessation among pregnant women, especially those who are underweight and in the older age groups, because of the increased risk of delivering premature babies.

\section{VITAMIN D LEVELS IN NEAR TERM INFANTS}

doi:10.1136/archdischild-2012-302724.1319

'W Al-Harthy, ${ }^{2} \mathrm{D}$ El-Metwaly, ${ }^{3} \mathrm{MM}$ Abou Al-Seoud. 'Neonatal Division, Paediatric Department, Maternity and Children Hospital, Makkah; Saudi Arabia; '2Paediatric, Suez Canal University (FOMSCU), Ismaillia, Egypt; ${ }^{3}$ Neonatal Division, Paediatric Department, King Fahad Armed Forces Hospital, Jeddah, Saudi Arabia

Background and Aims Based on the fact that the whole-body bone mineral content increases between 32-33 wk and 40-41 wk gestation (3.0 fold increase), near-term infants are thus at higher risk for low levels of vitamin D. Our aim was to measure 25-hydroxyvitamin D [25(OH) D] concentrations in the cord blood of near term infants born at KFAFH in Jeddah Saudi Arabia.

Methods This was a prospective study carried out from August $1^{\text {st }}$ 2011 to January $31^{\text {st }} 2012$. A total of 153 near terms babies with gestational ages 34 to $36+6$ weeks were included. The results were classified as Deficient $(25(\mathrm{OH}) \quad \mathrm{D}<37.5 \mathrm{nmol} / \mathrm{L})$, Insufficient $(25(\mathrm{OH}) \mathrm{D} 37.5-75 \mathrm{nmol} / \mathrm{L})$ or Sufficient $(25(\mathrm{OH}) \mathrm{D}>75 \mathrm{nmol} / \mathrm{L}]$.

Results The measured 25(OH) D levels ranged between 3-91 $\mathrm{nmol} / \mathrm{L}$ with a mean of $26.91 \pm 13.934 \mathrm{nmol} / \mathrm{L}$. The values were classified as deficient in 134 babies (88.2\%) and insufficient in 16 babies $(10.5 \%)$, while only two babies $(1.3 \%)$ had sufficient levels of vitamin $\mathrm{D}$ in their cord blood. We ran correlation studies between $25(\mathrm{OH})$ D levels, birth weight $(\mathrm{BWt})$, gestational age (GA) and admission to the neonatal intensive care (NICU), there was a weak correlation between $25(\mathrm{OH})$ D levels and birth weight $r$ value of. 172 $(p<0.05)$ but no correlation was found with GA or NICU admission.

Conclusion We observed a high prevalence of significant hypovitaminosis $\mathrm{D}$ among near term infants and a weak correlation with lower birth weight, the magnitude of which warrants identification and intervention.

\section{BIRTH AND DISCHARGE WEIGHT OF INFANTS WITH BIRTH WEIGHT LESS THAN $1000 \mathrm{G}$}

doi:10.1136/archdischild-2012-302724.1320

1J Tołloczko, 'A Góralska, ${ }^{2} \mathrm{~A}$ Borowiec, 'MK Kornacka. 'Department of Neonatology and Neonatal Intensive Care; ${ }^{2}$ Students Scientific Association of Neonatology, Medical University of Warsaw, Warsaw, Poland

Background and Aims Despite sweeping progress in the area of neonatology it is impossible to create artificial environment which would provide the same development conditions as mother's womb. Correct subsequent development of a child is dependant on intrauterine growth. Premature delivery hampers not only the development of internal organs but also weight gain. Aim of the project is to compare birth and discharge weight of infants with ELBW in order to analyse their weight gain.

Methods Data was gathered from 81 infants of weight less than $1000 \mathrm{~g}$ hospitalised in Neonatology and Infant Intensive Care Centre at the Medical University of Warsaw. Employing growth charts to compare birth and discharge weight of infants with ELBW. Assessing the influence of pregnancy length and sex of the infant on its weight gain.

Results The 81 infants were divided into 3 groups. Group 1 with 22 infants $(27.2 \%)$ whose weight-for-age score was the same for both birth and discharge weight. Group 2 with 53 infants (70.4\%) for which weight-for-age score lowered after birth. Group 3 with 6 infants $(2.4 \%)$ for which weight-for-age score increased. A statistically significant correlation was discovered between pregnancy length and weight gain rate in groups 1 and 2. No correlation was found between sex of the infant and its gain weight in any of the groups.

Conclusions For infants with ELBW postnatal weight gain does not match intrauterine growth. An important factor hampering postnatal weight gain is pregnancy length. The lower intrauterine age the slower weight gain rate.

\section{THE MANAGEMENT OF CENTRAL DIABETES INSIPIDUS IN NEONATAL INTENSIVE CARE UNIT: EXPERIENCE OF EIGHT CASES}

doi:10.1136/archdischild-2012-302724.1321

'S Alan, 'A Kılıç, 'U Çakır, 'D Yıldız, 'D Kahvecioğlu, ${ }^{2} \mathrm{M}$ Berberoğlu, ${ }^{2} Z$ Şıklar, ${ }^{2} \mathrm{G}$ Öçal, 'Ö Erdeve, 'B Atasay, 'S Arsan. 'Department of Pediatrics, Division of Neonatology; ${ }^{2}$ Department of Pediatrics, Division of Pediatric Endocrinology, Ankara University, Ankara, Turkey

Neonatal central diabetes insipidus (DI) is extremely rare and etiology has not been documented extensively. Asphyxia, intraventricular hemorrhage, severe infections, and central nervous system abnormalities have been associated with central DI in neonatal intensive care units (NICU). Desamino-8-D-arginine vasopressin (DDAVP) has been in clinical use for the treatment of central DI DDAVP preparations are available for intranasal, oral, subcutaneous, and intravenous administration. There is not clear data for the management and used DDAVP form for the central DI in neonates. In this article, we presented eight cases with different etiology of 